ISSN electrónico: 2602-8069

\title{
COMUNICACIÓN GESTUAL Y GESTOS DE AUTOCONTACTO EN LOS COMERCIANTES DEL CENTRO COMERCIAL "SIETE COLINAS" DE GUARANDA
}

\section{Gestual communication and self-contained gestures in the merchants of the "Siete Colinas" shopping center of Guaranda}

Elsita Margoth Chávez García Universidad Estatal de Bolívar, Carrera de Mercadotecnia emchavez@ueb.edu.ec

Charles Paul Viscarra Armijos

Universidad Estatal de Bolívar, Carrera de Mercadotecnia chviscarra@ueb.edu.ec

Liseth Nataly Vergara Hurtado

Universidad Estatal de Bolívar, Carrera de Mercadotecnia Ivergarahurtado@gmail.com

Zaida Margarita Sánchez Salazar

Universidad Estatal de Bolívar, Carrera de Mercadotecnia margaritazaidasanchez1998@gmail.com

Giovanny Lennin Haro Sosa

Escuela Superior Politécnica de Chimborazo, Carrera de Gastronomía y Promoción y Cuidados de la Salud gharo@espoch.edu.ec

Fecha de recepción del artículo: 23/04/2019

Fecha de aceptación definitiva: 18/06/2019 


\section{RESUMEN}

El trabajo de investigación se planteó como un análisis de la comunicación y los gestos de autocontacto en los comerciantes del Centro Comercial "Siete Colinas" en la ciudad de Guaranda en el período octubre 2018-febrero 2019". Se diseñó como objetivo general determinar la influencia de la comunicación y gestos de autocontacto en el comportamiento de los comerciantes y consumidores a través de conceptualizar las variables involucradas, identificar el tipo de comunicación que se aplica en el proceso de comercialización y así mejorar el mismo. La conceptualización de la temática deja ver la importancia que tiene el tema en el diario vivir de los comerciantes, quienes no conocen la influencia de una buena comunicación sin exceder en gestos de autocontacto y una efectiva comunicación gestual para fidelizar a los clientes sobre la oferta de servicios y productos. Para conocer dichas deficiencias y fortalezas se utilizó una metodología de investigación aplicada, no experimental y bibliográfica apoyada por la aplicación de encuestas a los dueños de los locales, de quienes se obtuvo información relevante para emitir como conclusión que la comunicación gestual es parte importante en el proceso de compra y venta entre comerciantes y clientes, puesto que determina en la mayoría de los casos la confianza que se genera entre ellos para efectivizar la misma y así fidelizar a los clientes.

Palabras clave: Comunicación no verbal, atención al cliente, satisfacción del cliente, comportamiento del consumidor.

\section{ABSTRACT}

This research work was considered as an analysis of communication and self-contact gestures, in the merchants of the Shopping Center "Siete Colinas" in the city of Guaranda in the period October 2018 - February 2019". It was designed as a general objective to determine the influence of communication and self-contact gestures on the behavior of traders and consumers through conceptualizing the variables involved, identifying the type of communication, which is applied in the commercialization process and thus improving the same. The conceptualization of the theme reveals the importance of the topic in the daily lives of the merchants, who do not know the influence of good communication without exceeding in self-contact gestures and an effective gestural communication to make clients loyal about the offer of services and products. In order to know these deficiencies and strengths, a methodology of non-experimental and bibliographic research was applied, supported by conducting surveys to the owners of the premises, from which relevant information was obtained to conclude that gestural communication is an important part of the process of buying and selling between merchants and customers, as it determines in most cases the trust that is generated between them to make it effective.

Keywords: Non-verbal communication, customer satisfaction, customer support, consumer behavior. 


\section{INTRODUCCIÓN}

En la actualidad es trascendental conocer y dar importancia a la comunicación, sobre todo a la comunicación gestual y los gestos de autocontacto en la parte interpersonal, en especial cuando se entablan negocios en una cultura en la que la confianza es la base de las relaciones a largo plazo. Sobre todo considerando que la gestualidad actúa en forma persuasiva ante el receptor, al igual que el no verbalismo, que forma parte de la comunicación y cumple el mismo papel en el proceso de ventas.

En el ámbito popular las formas de expresión son una de las más influyentes, considerando los aspectos culturales de los pueblos y sociedades, y aquellos individuales en los que el hombre comunica con sus actitudes, gestos, ojos, movimiento del cuerpo o a través de diversas expresiones de su rostro (Soto, 2015).

En la comunidad del grupo de comerciantes del centro comercial "Siete Colinas", objeto de esta investigación, se desconoce, tanto manera formal como académica, sobre la influencia de la comunicación, sea esta gestual, y la de autocontacto en función del servicio, produciéndose a veces equivocaciones involuntarias entre ellos que afectan las ventas de una u otra forma.

El Centro Comercial "Siete Colinas" está ubicado en el sector del Barrio 15 de Mayo, en los bajos de la Unidad Educativa Pedro Carbo, en la ciudad de Guaranda (Hurtado, 2018). Fue creado en el año 2000 durante la alcaldía del Sr. Alberto Coles, quien tenía como fin entregar a la comunidad un lugar adecuado en donde promocionar las ventas y realizar el comercio de forma adecuada y ordenada. Actualmente, la administración está a cargo del Distrito de Educación de Bolívar, entidad que no cumple mayormente las funciones que conlleva y solo se limita a recaudar el dinero que generan los arriendos.

Cuenta con 17 puestos de comercio que ofrecen diversos servicios y productos, entre ellos: peluquería, costura, copiadoras, asesorías de proyectos, acceso a plataformas de instituciones educativas, capacitaciones a cursos de nivelación, diseños de carteleras. Existen también locales destinados a la venta de comida rápida, productos ortopédicos, ropa, artículos de bazar y papelería, trajes étnicos, entre otros.

La parte comercial, entendida como el espacio para la compra y venta de bienes y servicios, se complementa con la parte expresiva (comunicación verbal y no verbal), analizada desde el punto de vista comunicacional, y clasificada por las expresiones gestuales y de autocontacto como: el saludo, que en ocasiones es frío y sin dar importancia al cliente; los movimientos corporales, que denotan desgano y poco interés en realizar la venta; la no adecuada manipulación del objeto de venta; expresiones faciales que pueden transmitir emociones y sentimientos poco agradables, los cuales perjudican en gran medida las relaciones existentes entre clientes y comerciantes.

En el trabajo de investigación titulado "Los efectos persuasivos de la gestualidad en los discursos mediáticos" (Georget, 2009) se estudia el rol persuasivo de los gestos en la comunicación mediática y del receptor, considerando al no verbalismo como un índice periférico en el tratamiento de las comunicaciones persuasivas.

En el documento "Contradicciones entre el lenguaje verbal y no verbal del discurso político. Aproximación teórica para analizar la comunicación no verbal de un personaje en distintos contextos" (Manfredi y García, 2010) se concluyó que la comunicación no verbal puede mostrar aspectos contradictorios con el discurso verbal. Algunas expresiones corporales se mantienen en distintos contextos, habituales de cada persona, que varían de acuerdo al escenario en donde se producen. 
Setó (2003), en su trabajo sobre la fidelidad del cliente en el ámbito de los servicios, señala que al analizar la relación entre variables como la calidad de servicio con la fidelidad del cliente —entendida esta como una intención de comportamiento- estas se centran únicamente en la dimensión fidelidad, ya que además de ser la que presenta una mayor fiabilidad es la que mejor capta la esencia por la calidad de servicio, la satisfacción, la imagen o la confianza.

Esta concepción de la comunicación no verbal y la posible presentación de aspectos contradictorios al momento de establecer la comunicación entre vendedor y cliente puede ser negativa o positiva de acuerdo con la habilidad que posee el primero para expresarse e interactuar con los segundo, sobre todo tomando en cuenta que el proceso comunicativo implica la emisión de señales (sonidos, gestos, señas) con la intención de dar a conocer un mensaje y que para que la comunicación sea exitosa, el receptor debe contar con las habilidades que le permitan interpretar el mensaje (Porto, 2008).

Según Martínez y otros (2014), la comunicación es un fenómeno inherente a la relación que los seres vivos mantienen cuando se encuentran en grupo. A través de la comunicación las personas obtienen información respecto a su entorno y pueden compartirla con el resto. Sin embargo, para Moncada (2015) la comunicación cumple un rol fundamental en este contexto, pues se expresa en estas relaciones, hechos, mundos e intercambios simbólicos, prácticas sociales y narraciones inmersas en el sistema-mundo capitalista que Occidente ha creado, aplicando en esta tesis dos dimensiones de comunicación: producción/ consumo de mercancías musicales y lenguaje en narraciones de canciones.

Al considerar que la comunicación gestual o no verbal sirve para afirmar, contradecir complementar o reforzar todos los tipos de comunicación, se debe tomar también en cuenta que dentro del lenguaje no verbal se consideran tres grupos de señales denominadas, quinesia, proxémica y paralingüística, las cuales se refieren al movimiento del cuerpo, al espacio o distancia que establecen y a las características sonoras y temporales (volumen, intensidad, velocidad, vocalización, uso de las pausas) que forman parte de la conducta verbal, respectivamente (Didáctica Educación, 2019).

La proxémica, según Muelas (2018), es el estudio de las relaciones de proximidad y alejamiento entre personas y objetos durante las interacciones; cabe señalar que cada cultura establece el tipo de contacto. Las culturas de alto contacto son aquellas donde las distancias entre las personas tienden a ser menores, mientras que en las culturas con bajo contacto el espacio entre personas que interactúan es mucho mayor.

El espacio alrededor de una persona puede dividirse en cuatro distancias zonales bien claras:

- Zona íntima (de 15 a $45 \mathrm{~cm}$ ). Es la más importante y es la que una persona cuida como un espacio propio al que solo se le permite la entrada a quienes están muy ligados a la de manera emocional.

- Zona personal (entre $46 \mathrm{~cm}$ y $1.22 \mathrm{~m}$ ). Es la distancia que separa a las personas en una reunión social o en el espacio dentro de la oficina, y en las fiestas.

- Zona social (entre 1,22 y 3,6 metros). Es la distancia que nos separa de la gente que no conocemos bien.

- Zona pública (a más de $3.6 \mathrm{~m}$ ). Es la distancia cómoda para dirigirnos a un grupo de personas (Ferrari, 2018).

Se hace necesario definir y expresar el significado de los deslices gestuales, los cuales, según Emily (2009), son los fallos de la máscara facial, porque cuando se distorsio- 
na la verdad los gestos faciales cambian inmediatamente. Estos presentan las categorías siguientes:

- Gestos faciales. El rostro cambia todos los gestos.

- Movimiento de las manos. Las manos o se mueven mucho o están totalmente pasivas. Esto puede ser una señal de que la persona está mintiendo.

- Movimiento de piernas. Cuando se miente, se mueve las piernas demasiado o se las mantiene estáticas por un periodo prolongado.

- El efecto espejo. Nos hace parecernos a la persona que tenemos en frente; por eso nos sentimos cómodos con quien se parece a nosotros (Martínez y Imanaka, 2014).

"Las habilidades comunicativas para transmitir la información de una manera asertiva y la capacidad persuasiva para convencer a los clientes de lo que se considera conveniente son requisitos necesarios para toda relación con el cliente" (Ongallo, 2012). También se las conoce como la razón o la fuerza que empuja al individuo al consumo de un producto o servicio por un estado de tensión, resultado o necesidad no satisfecha, lo que puede provocar en el individuo conductas orientadas a satisfacer sus necesidades o reducir la tensión que siente (Escudero, 2011)

Se puede acotar que la forma de comunicación del vendedor tendrá un efecto en el comportamiento del consumidor, el cual se enfoca en la forma en que los individuos toman decisiones para gastar sus recursos disponibles (tiempo, dinero y esfuerzo) en artículos relacionados al consumo. Esto incluye el por qué compran, cuándo, dónde, con qué frecuencia, cuán a menudo usan el producto, cómo lo evalúan después de la compra y cuál es la influencia de tal evaluación con compras futuras o cómo lo desechan (Kanuk y Schiffman, 2010).

Se puede recibir retroalimentación en relación con la satisfacción del cliente a través de reclamos sobre los vendedores o productos, los cuales pueden revertirse en programas de mejora de calidad y servicio en la venta directa.

Una parte importante de la actividad de quien está a cargo de la venta directa es informar al cliente, dar las indicaciones y prescripciones necesarias y responder las inquietudes que surjan sobre el producto; para ello el vendedor debe poseer conocimientos suficientes sobre el bien o servicio que ofrece y suficiente experiencia para ser competitivo en el campo de la comunicación humana y así alcanzar las metas propuestas (Ongallo, 2012).

Las relaciones que se producen entre los clientes y los vendedores están dadas bajo ciertos aspectos propios del intercambio de necesidades. Entre ellos se destacan varios aspectos como:

- Los proveedores de servicios pueden crear mayores vínculos de fidelidad con sus clientes que los proveedores de bienes tangibles.

- La fidelidad es más fuerte entre los consumidores de servicios que entre los consumidores de bienes.

- Los servicios brindan más oportunidad de interacciones personales, lo que permite fomentar de mejor manera la fidelidad.

- En algunos casos cambiar de proveedores de servicios representa mayor riesgo que cuando se cambia un proveedor de bienes (Setó, 2003).

Para Kanuk y Schiffman (2010) "el consumidor personal compra bienes y servicios para su consumo propio", pues la mayor parte de estas compras son para su familia, amigos o para al hogar. Mientras que el consumidor organizacional "lo constituyen las empresas 
con propósitos de lucro o sin ello, dependencias gubernamentales e institucionales y todos quienes compran productos, bienes y servicios para su desempeño".

\section{METODOLOGÍA}

La presente investigación contempla un enfoque mixto, cualitativo y cuantitativo. Desde la perspectiva cuantitativa se realizó una encuesta, los datos obtenidos fueron representados por medio de cuadros y gráficos estadísticos. Para Monje (2017) la investigación científica, desde el punto de vista cuantitativo, se trata de un proceso sistémico y ordenado que se lleva a cabo siguiendo determinados pasos. Planear una investigación consiste en proyectar el trabajo de acuerdo con una estructura lógica de decisiones y con una estrategia que oriente la obtención de respuestas adecuadas a los problemas de indagación propuestos.

Desde la perspectiva cualitativa, mediante un proceso de observación se obtuvo las características de los procesos de comunicación y comunicación gestual que se producen entre los vendedores y clientes, los cuales, según Salgado (2007), son más flexibles y abiertos, y el curso de las acciones se rigen por el campo (los participantes y la evolución de los acontecimientos). De este modo el diseño se va ajustando a las condiciones del escenario o ambiente.

Para abordar la investigación cualitativa y cuantitativamente se utilizó el método deductivo, que es un sistema para organizar hechos conocidos y extraer conclusiones, lo cual se logra mediante una serie de enunciados obteniendo información de casos y hechos particulares o situaciones que se dan en cada local comercial para poder establecer una o varias conclusiones como resultado de la investigación (Dávila, 2006).

El lugar que se destinó para la investigación fue el Centro Comercial "Siete Colinas", ubicado en la parroquia Gabriel Ignacio Veintimilla del cantón Guaranda, provincia de Bolívar, en donde se aplicaron encuestas a los dueños de cada uno de los locales comerciales, con base en preguntas cerradas y de opción múltiple, diseñadas a partir de las variables identificadas: comunicación gestual y gestos de autocontacto, y el comportamiento de los comerciantes y consumidores como variables independiente y dependiente, respectivamente. También se utilizó la observación como una técnica que para permite conocer la realidad mediante la percepción directa de los objetos y fenómenos (eumed.net, 2012).

Por decisión de los investigadores no se aplicó ninguna fórmula de muestreo para las encuestas, ya que la población es finita y el número total de personas es manejable para la indagación; esto es, 17 personas encargadas de los locales comerciales del Centro Comercial "Siete Colinas". Para aplicar la observación como una técnica se realizó un muestro por conveniencia, en el cual los investigadores registraron información diaria en una ficha en diversos momentos del proceso de venta.

\section{RESULTADOS}

La población total investigada fue de 17 personas que laboran en los locales del Centro comercial "Siete Colinas" de forma permanente.

Al preguntar a los encuestados sobre la forma más utilizada de comunicación se obtuvo los siguientes resultados (tabla 1): 
COMUNICACIÓN GESTUAL Y GESTOS DE AUTOCONTACTO EN LOS COMERCIANTES DEL CENTRO COMERCIAL "SIETE COLINAS" DE GUARANDA

Tabla 1. Comunicación más utilizada

\begin{tabular}{lcc}
\hline \multicolumn{1}{c}{ Alternativas } & Frecuencia & Porcentaje (\%) \\
\hline Comunicación verbal & 13 & 76 \\
\hline Comunicación gestual & 4 & 24 \\
\hline Total & 17 & 100 \\
\hline
\end{tabular}

Fuente: Dueños de los locales del centro comercial 7 colinas.

Elaborado por los investigadores

La tabla anterior muestra que un $76 \%$ de personas recurren al tipo de comunicación verbal, mientras que el $24 \%$ la complementan con la comunicación gestual; en este sentido los empresarios interactúan con sus clientes principalmente a través de este tipo de comunicación verbal, debido a que encuentran más fácil tratar con los clientes de ese modo.

La siguiente pregunta se centró en conocer si los encuestados cuentan con un tipo de comunicación específica en el local comercial para los clientes (tabla 2):

Tabla 2. Comunicación específica

\begin{tabular}{|c|c|c|}
\hline Alternativas & Frecuencia & Porcentaje (\%) \\
\hline No & 12 & 71 \\
\hline Sí & 5 & $29 \%$ \\
\hline Total & 17 & 100 \\
\hline
\end{tabular}

Fuente: Dueños de los locales del Centro Comercial "Siete Colinas".

Elaborado por los investigadores

El $71 \%$ de los encuestados no cuenta en su local con ningún tipo de comunicación visual para sus clientes; el $29 \%$ tienen algún tipo de comunicación visual como la señalética para salida de emergencia, ECU 911, servicios higiénicos o letreros publicitarios, entre otros.

Para la siguiente pregunta, se buscó conocer si los propietarios consideran que la comunicación utilizada es adecuada para sus clientes.

Tabla 3. Adecuada comunicación

\begin{tabular}{lccc}
\hline & Alternativas & Frecuencia & Porcentaje (\%) \\
\hline Sí & 15 & 88 \\
\hline No & 2 & 12 \\
\hline Total & 17 & 100 \\
\hline
\end{tabular}

Fuente: Dueños de los locales del Centro Comercial "Siete Colinas".

Elaborado por los investigadores 
Aquí el $12 \%$ de los encuestados manifiesta que el tipo de comunicación que utilizan no es el adecuado para sus clientes y el $88 \%$ afirma que sí lo es. Esto demuestra que la mayoría atienden a sus clientes bajo modalidades verbales y gestuales, aunque ciertos comerciantes no utilizan algún tipo de comunicación formal y afirman que sus clientes ya están acostumbrados a ese trato.

También se preguntó si consideran que la comunicación gestual les ayudaría a tener un mejor desenvolvimiento con los clientes, en lo cual respondieron (tabla 4):

Tabla 4. Desenvolvimiento con los clientes

\begin{tabular}{|l|c|c|}
\hline \multicolumn{1}{|c|}{ Alternativas } & Frecuencia & Porcentaje \\
\hline $\mathrm{Si}$ & 17 & $100 \%$ \\
\hline No & 0 & $0 \%$ \\
\hline Total & 17 & $100 \%$ \\
\hline
\end{tabular}

Fuente: Dueños de los locales del Centro Comercial "Siete Colinas"

Elaborado por los investigadores

El total de encuestados considera que la comunicación gestual les ayudaría a desenvolverse mejor con sus clientes. Sobre todos para quienes conocen los beneficios de la comunicación gestual, aunque dicho conocimiento sea empírico y parta de las experiencias vividas en el día a día con el servicio que dan a sus clientes.

Finalmente, se recabó información acerca de las personas que estarían dispuestos a utilizar una serie de deslices gestuales (gráfico 1).

Gráfico 1. Deslices gestuales

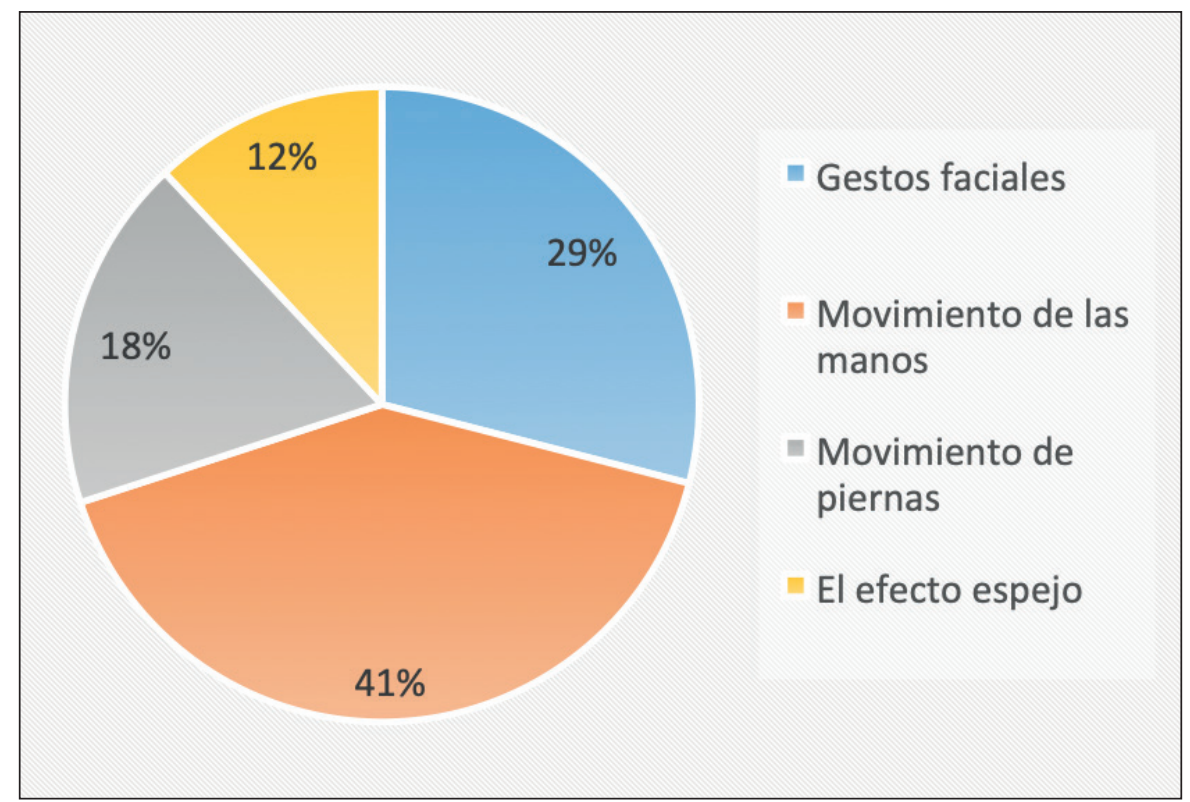

Fuente: Dueños de los locales del Centro Comercial "Siete colinas".

Elaborado por los investigadores 
Se conoce también que, del total de encuestados, el $41 \%$ se inclina a utilizar el movimiento de manos como un desliz gestual con el cliente, el $29 \%$ usaría gestos faciales, el $18 \%$ lo haría con el movimiento de las piernas y el $12 \%$ con el efecto espejo. Estos deslices gestuales son formas comunes de expresión entre los comerciantes encuestados, pues son prácticas de comunicación que les permiten establecer confianza y amabilidad entre ellos.

De la misma manera, se puede expresar que el total de las personas indagadas están dispuestas a acudir a charlas o talleres para mejorar la atención al cliente.

Para complementar la investigación se utilizó una ficha de observación como un instrumento de recolección de información directa, en donde se pudo registrar el desenvolvimiento de los dependientes hacia los clientes.

En la siguiente tabla se exponen los resultados de una manera sintetizada, en la cual se registró datos diarios, con una frecuencia de 5 días.

Tabla 5

\begin{tabular}{|c|c|c|c|}
\hline \multicolumn{4}{|c|}{ Ficha de observación: 1, 2, 3, 4,5} \\
\hline Elaboración & \multicolumn{3}{|c|}{ Los investigadores } \\
\hline Lugar & \multicolumn{3}{|c|}{ Centro Comercial "Siete Colinas" } \\
\hline \multirow{5}{*}{ Fecha } & $5-06-2019$ & \multirow{5}{*}{$\begin{array}{l}\text { Características } \\
\text { observadas }\end{array}$} & Poco contacto visual con el cliente \\
\hline & $6-06-2019$ & & $\begin{array}{l}\text { Ausencia de contacto visual con el cliente } \\
\text { Cliente sigue visualmente al dependiente }\end{array}$ \\
\hline & $7-06-2019$ & & $\begin{array}{l}\text { Ausencia de gestos con las manos } \\
\text { Conversación entre dependientes de locales } \\
\text { comerciales a una distancia prolongada y con } \\
\text { tono de voz alto }\end{array}$ \\
\hline & 8-06-2019 & & $\begin{array}{l}\text { Antes de dar una opción el dependiente se } \\
\text { rascaba la cabeza con frecuencia }\end{array}$ \\
\hline & $9-06-2019$ & & $\begin{array}{l}\text { El cliente preguntó sobre un producto y le die- } \\
\text { ron una respuesta negativa, sin otras opcio- } \\
\text { nes o recomendaciones } \\
\text { Dependiente no se levantó de la silla para } \\
\text { atender al cliente } \\
\text { Uso prolongado del celular }\end{array}$ \\
\hline \multirow{5}{*}{$\begin{array}{l}\text { Duración } \\
\text { cronometrada } \\
\text { de observación } \\
\text { in situ }\end{array}$} & \multirow{5}{*}{$\begin{array}{l}60 \text { minutos } \\
\text { diarios }\end{array}$} & \multirow{5}{*}{$\begin{array}{l}\text { Duración promedio } \\
\text { de atención por } \\
\text { cliente }\end{array}$} & 15 minutos \\
\hline & & & 5 minutos \\
\hline & & & 30 minutos \\
\hline & & & 10 minutos \\
\hline & & & 8 minutos \\
\hline
\end{tabular}

Elaborado por los investigadores

Para complementar la información, se puede decir que mientras se realizaba la indagación in situ, los dependientes de los locales comerciales manifestaron que muchas veces han perdido clientes por sus formas de expresión, tanto verbal como no verbal, y que a largo 
plazo esto ha repercutido en la reputación de cada uno de ellos, siendo un determinante específico para el éxito o fracaso de los emprendimientos presentes en el Centro Comercial "Siete Colinas".

\section{CONCLUSIONES}

La investigación realizada constituye un aporte de gran importancia para los negociantes del centro comercial en cuestión, ya que los resultados obtenidos permitieron comprender de mejor manera la relación de comunicación, comunicación gestual y gestos de autocontacto que puedan ayudar en mejorar las ventas y las relaciones interpersonales.

El interés radica en lo novedoso del tema, ya que se descubren las debilidades de los comerciantes al realizar las ventas utilizando algunas formas de comunicación como la gestualidad y el autocontacto como principales herramientas. También se conoce que la indagación es original porque parte de una necesidad local que surge en un lugar conocido y que no ha sido identificado como objeto de estudio por otros investigadores.

La pertinencia de esta investigación, además de que cuenta con el apoyo de los comerciantes del lugar, es el interés que encuentran los entrevistados en identificar las deficiencias en el proceso de venta para mejorar su nivel económico, de comercialización y para brindar un mejor servicio a la comunidad.

Los beneficiarios directos son los comerciantes del centro comercial, ya que en conjunto se podrán identificar sus debilidades en la comunicación con sus clientes y la forma como mejorar para prestar un mejor servicio y así promocionar e incrementar las ventas en sus negocios, así como la fidelización de sus clientes.

Los resultados obtenidos en la investigación permitieron conocer las características de la comunicación gestual y los gestos de autocontacto con la finalidad de cambiar el comportamiento de los comerciantes en función de los clientes y mejorar así las relaciones económicas y financieras de las familias.

Luego de haber determinado que la problemática se centra en el poco contacto visual, ausencia de una atención real, presencia de varios elementos que producen ruido comunicacional, gestos inapropiados, uso del celular durante el proceso de ventas, entre otras, se puede exhortar a los dependientes para mejorar sus relaciones a largo plazo con los consumidores y ofrecer una eficiente atención y servicio hacia el cliente, acompañado de una excelente comunicación integral.

Entre otras características, la postura corporal, los gestos, la mirada, la sonrisa, el equilibrio del temperamento deben mantenerse de una manera sutil, natural y espontánea para poder acompañar a las palabras con el cuerpo, la postura y los movimientos utilizados al momento de expresarse.

En el entorno comercial, la comunicación verbal y no verbal es un recurso importante, convirtiéndose en una estrategia para poder cerrar una venta y mantener relaciones a largo plazo con los clientes, ya que esta enfatiza, contextualiza y determina lo que queremos que la otra parte entienda.

Lo que se logrará es que los propietarios de los locales del centro comercial "Siete Colinas" generen mayores relaciones a largo plazo con los clientes, sean un referente de atención y servicio para sus compradores y, sobre todo, que mediante la efectiva comunicación generen mayor sustentabilidad y minimicen el riesgo de fracaso de sus emprendimientos.

El sustento teórico y la información obtenida a través del aporte de los comerciantes encuestados han permitido concluir que la comunicación gestual forma parte importante en 
el proceso de compra y venta entre comerciantes y clientes, pues determina, en la mayoría de los casos, la confianza que se genera entre ellos para efectivizar la misma; en especial si se toma en cuenta que los comerciantes conocen y practican la comunicación gestual, de auto contacto y no verbal a través de prácticas diarias en la atención al cliente, sin que lo hayan visto como una disciplina de estudio.

Los comerciantes del Centro Comercial "Siete Colinas" deben mejorar las formas de comunicación, comunicación gestual, de autocontacto y demás formas de relacionarse con los clientes para satisfacer no solo sus necesidades de consumo sino las relaciones interpersonales entre las dos partes.

Para lograr la eficiente comunicación integral deseada se requiere capacitar a los comerciantes investigados para que se preparen y mejoren las estrategias de comunicación gestual, de autocontacto y no verbal en las prácticas diarias y de atención al cliente.

Finalmente, se determina que la comunicación efectiva, sea esta gestual, de autocontacto u otras, que se definen como estrategias viables para la transmisión de información, debe generar confianza y seguridad al realizar las ventas, lo que demuestra una completa habilidad en las relaciones interpersonales.

\section{BIBLIOGRAFÍA}

Asamblea Constituyente. (2012). Constitución del Ecuador. Obtenido de https://www.google.com/url? sa=tyrct=jyq=yesrc=sysource=webycd=11ycad $=$ rjayuact $=8 \mathrm{yved}=2$ ahUKEwjOm7SagPXeA hUQjlkKHYuzCqMQFjAKegQICRACyurl=https\%3A\%2F\%2Fwww.oas.org\%2Fjuridico\%2Fp dfs\%2Fmesicic4_ecu_const.pdfyusg=AOvVaw0sQShi2LIw-MyD2IVirbGH

Bibliotecas Duoc UC. (01 de 01 de 2018). Bibliotecas Duoc UC. Obtenido de http://www.duoc.cl/biblioteca/crai/definicion-y-proposito-de-la-investigacion-aplicada

Congreso Nacional. (2011). Ley Orgánica de Defensa del Consumidor. Obtenido de https://www. industrias.gob.ec/wp-content/uploads/2015/04/A2-LEY-ORGANICA-DE-DEFENSA-DELCONSUMIDOR.pdf.

Dávila, G. (2006). El razonamiento inductivo y deductivo dentro del proceso investigativo en ciencias experimentales y sociales. Laurus. ISSN 1315-883X, 27.

Didáctica Educación. (01 de 01 de 2019). Obtenido de https://rodas5.us.es/file/ed60c2a2-ee9e-666a188d-189a4ffe9042/1/capitulo5_SCORM.zip/pagina_08.htm

Emily. (25 de noviembre de 2009). blogger.com. Obtenido de COMPORTAMIENTO DE AUTOCONTACTO: http://emilua3008.blogspot.com/2009/11/comportamiento-de-autocontacto.html

Escudero, J. (2011). Gestión comercial y servicio de atención al cliente. Madrid, España: Editorial Paraninfo. eumed.net. (01 de 01 de 2012). Enciclopedia virtual. Obtenido de Técnicas e instrumentos de investigación: http://www.eumed.net/tesis-doctorales/2012/mirm/tecnicas_instrumentos.html

Ferrari, L. (2018). Cómo conocer a las personas por su lenguaje corporal. Madrid, España: Tus buenos libros.

Georget, P. (2009). Los efectos persuasivos de la gestualidad en los discursos . Obtenido de Instituto de la Comunicación e Imagen. Universidad de Chile: https://docplayer.es/51886941Los-efectos-persuasivos-de-la-gestualidad-en-los-discursos-mediaticos-1-the-persuasiveeffects-of-gestures-in-media-discourse.html

Hurtado, L. (23 de noviembre de 2018). Historia del Centro Comercial Siete Colinas. (L. Vergara, Entrevistador)

Kanuk, H., y Schiffman, L. (2010). Comportamiento del consumidor. Barcelona, España: Ediciones Pearson.

Manfredi, J., y García, Á. (2010). Contradicciones entre el lenguaje verbal y no verbal del discurso político. Aproximación teórica para analizar la comunicación noverbal en distintos contextos. 
Obtenido de Universidad de Sevilla. Máster en Comunicación Institucional y Política: http:// nardonecustombuilders.com/59570055-Test-de-comunicacion-1.html

Martínez, C., y Imanaka, A. (2014). Cómo influye el lenguaje corporal en el proceso de venta. Obtenido de https://www.contunegocio.es/marketing/como-influye-el-lenguaje-corporal-en-elproceso-de-venta/

Moncada, N. (01 de 01 de 2015). Flacso. Obtenido de file://C:/Users/elmar/Downloads/TFLACSO2015NRML.pdf

Monje, C. (01 de 02 de 2017). Guía didáctica metodología de la investigación cuantitativa y cualitativa. Obtenido de https://www.uv.mx/rmipe/files/2017/02/Guia-didactica-metodologia-de-lainvestigacion.pdf

Muelas, R. (30 de 01 de 2018). La mente es maravillosa. Obtenido de https://lamenteesmaravillosa. com/proxemica-comunicar-espacio/

Ongallo, C. (2012). La atención al cliente y el servicio posventa. Madrid, España: Díaz de Santos.

Porto, J. (2008). DEFINICION.DE. Obtenido de Comunicacion: https://definicion.de/comunicacion/

Salgado, A. (2007). Investigación cualitativa: diseños, evaluación del rigor metodológico y retos. Liberabit. Revista de Psicología 13: 71-78.

Setó, P. (2003). La fidelidad del cliente en el ámbito de los servicios; una análisis de la escala "Intenciones de comportamiento". Universidad Rovira I Virgili. Investigaciones Europeas de Dirección y Economía de la Enpresa.

Soto, M. (01 de 01 de 2015). Obtenido de https://dspace.ups.edu.ec/bitstream/123456789/9787/1/ QT08020.pdf

Vargas, Z. (2009). La investigación aplicada: una forma de conocer las realiades con evidencia científica. Revista Educación 33(1), 155-165. 


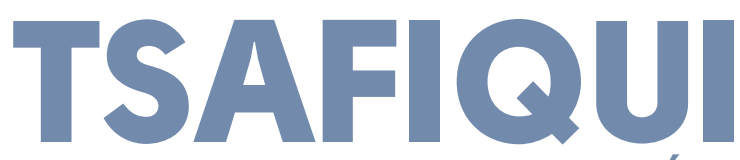
REVISTA DE INVESTIGACIÓN CIENTÍFICA 